\title{
Evidence-based medicine or patient-centered medicine, or both?
}

We are in an era of medicine where biological science, information, intolerance of uncertainty, commoditization, empathy scarcity, utilitarianism, and technology, among others, predominate. These aspects displace humanism, one of the pillars of medicine since time immemorial and, in this way, the focus of medicine is shifting further away from the patient.

Karl Jaspers, a physician and outstanding philosopher, left us the following quote more than 50 years ago, characterizing what was happening then: "In modern medicine, everything seems to be in the best of orders. Each day, great results are achieved. But the amazing thing is that, for patients and doctors, dissatisfaction increases." ${ }^{1}$

Dissatisfaction relentlessly increased in both groups, because doctors perceive that their profession has lost many of its values and people increasingly have less confidence in them. Likewise, patients are dissatisfied because clinical consultations are rushed and doctors are not empathetic and do not have an adequate clinical judgment; and to make this up they ask for ancillary studies that are mostly unnecessary.

Symptom management is the cornerstone of clinical care, especially for patients with chronic conditions. However, when taking care of patients, doctors do not detect their symptoms, physical disabilities, and concerns. In the USA, this happens in up to half of the clinical consultations, especially those that take place in healthcare centers. ${ }^{2}$

Probably, in order for medicine to improve, evidence-based medicine (EBM) emerged more than 25 years ago, placing randomized controlled trials (RCTs) at the top, above all other types of research. ${ }^{3}$ This methodology was used for the first time in 1948 when Doctor D'Arcy Hart and Sir Bradford Hill, of England, randomly assigned tuberculosis patients to two treatment arms, streptomycin and placebo. ${ }^{4}$ This study allowed streptomycin to save the lives of many patients by drastically changing survival in patients with tuberculosis.

With RCTs, a new era began together with evidence and high-quality observational studies, with healthcare providers' knowledge and experience. Undoubtedly, EBM has resulted in multiple benefits for patients through wellconducted, high-quality, and appropriate research studies in different populations, as observed in systematic reviews carried out in a satisfactory manner.

However, EBM has several weaknesses in relying openly on RCTs since most research is not tested in clinical settings and in heterogeneous populations. For instance, we see that, in practice, physicians run the risk of extrapolating estimates from a population to diagnose individual patients. "Many patients are similar, but all patients are different. Although two patients suffering from the same disease may present the same signs, symptoms, and identical laboratory tests, they will be two different patients" (Sacristan J. ${ }^{3}$ ). Something similar would be the old aphorism in medicine that says "there are no diseases, there are patients."

It is worth mentioning that the populations being studied are different than an individual patient, and therefore EBM raises the doubt whether RCTs results could guide the decisions in individual patients. Despite EBM successes, the significant variability in its implementation is still a serious problem.

However, there are other external factors that are increasing and clearly distort EBM founding principles, leading to a certain loss of prestige in recent years. Greenhalgh et al. have pointed out that there is a crisis because it has mainly been pervaded by commercial interests, especially from the pharmaceutical and medical device industries. The large bulk of evidence is already unmanageable and its quality has been monopolized and distorted by particular interests. Likewise, the industry sets the drug dose to be administered to the treatment and control groups, and publishes results in the main journals. According to Greenhalgh, EBM "has drifted in recent years from investigating and managing established disease to detecting and intervening in non-diseases." True evidence must be customized to the patient's needs and should not be restrained by rules and interests. ${ }^{5}$

It should also be noted that it is essential that evidence goes hand in hand with uncertainty, which is the only approach that will allow us to reflect on whether our clinical judgment is correct or wrong. Uncertainty is unavoidable when applying evidence resulting from RCTs conducted on populations but not on individuals.

Iona Heath underscored, in one of her essays, the need for physicians to know the evidence 
with clear indications of the limitations and that, through uncertainty, they are able to acknowledge potential harms; and added that "this would encourage clinicians to think instead of telling them what to do."

EBM makes us describe people in terms of biomedical science data, but these are not, and never will be, enough. Such evidence is essential, but always insufficient for the care of patients.

Abbasi, in an editorial called We need more humanity as well as better evidence, pointed out that our obsession with evidence may lessen our humanism in a clinical consultation, and quoted Iona Heath, who argued that "to provide consistent and better-balanced care, we need a new approach, so that doctors can bridge the gap between evidence and humanism."7

Limitations to the evidence base are clearly depicted in the study by Nagendran et al., who examined whether the significant effects of RCT results were replicated in followup studies. Unless the RCT effect size had a relative risk $\geq 20$, the follow-up study showed no significant effects. ${ }^{8}$

Regarding patient-centered medicine (PCM), it arose more than 20 years after EBM. The objective of this movement was to vindicate the need to look toward the individual patient, understood as a person. ${ }^{3}$ Throughout history, there have been many illustrious physicians who have advocated this view. It is very likely that William Osler, an exceptional clinician and great humanist, was the forerunner of what 90 years later those who designed the PCM movement would adopt. A wise sentence of the many that Osler left us and that supports PCM states "If it were not for the great variability among individuals, medicine might as well be a science and not an art." With these words he is emphasizing that art is essential in medicine and our variability makes this evidence often be insufficient to apply it without reflecting

Millenson pointed out that PCM was born as a conceptual framework that "consciously adopts the patient's perspective," and emphasized that currently, technological, economic, and social changes are moving healthcare in directions unanticipated by the patient centeredness pioneers. For Millenson, today PCM "is part of a larger set under the larger forces of economic interests, in an increasingly commoditized medicine where profit is constantly spreading." ${ }^{\prime \prime}$
As we see, both forms of medicine are deteriorating due to the harmful changes invading our profession with powerful profitbased interests.

However, there is currently an interest that EBM and PCM come together so that physicians and patients can evaluate research evidence and demand a more personalized medicine. This joint strategy could be beneficial to conduct research studies focused on meeting patients' needs and on a population-centered approach. ${ }^{3}$

It is likely that this is the only way to achieve a better outcome in healthcare and that investigations of evidence prevent problems that may harm patients, who obviously do not deserve them because, without any doubts, physicians should always watch over them.

\section{José María Ceriani Cernadas}

Editor

http: / / dx.doi.org/ 10.5546/ aap.2018.eng.90

To cite: Ceriani Cernadas JM. Evidence-based medicine or patientcentered medicine, or both? Arch Argent Pediatr 2018;116(2):90-91.

\section{REFERENCES}

1. Jaspers K. La práctica médica en la era tecnológica. Editorial Gedisa, Barcelona,1988.

2. Basch E. Patient-Reported Outcomes-Harnessing Patients' Voices to ImproveClinical Care. NEnglJMed 2017.376;2:106108.

3. Sacristán JA. Medicina basada en la evidencia y medicina centrada en el paciente: algunas reflexiones sobre su integración. RevClin Esp.2013;213:460-64.

4. Medical Research Council Streptomycin in Tuberculosis Trials Committee. Streptomycin treatment for pulmonary tuberculosis. BritMed J.1948;2:769--83.

5. Greenhalgh T, Howick J, MaskreyN. Evidence based medicine: a movement in crisis? BMJ 2014; 348:g3725.

6. Heath I. How medicine has exploited rationality at the expense of humanity: an essay by Iona Heath. BMJ 2016; 355:i5705.

7. Abbasi K. We need more humanity as well as better evidence. BMJ 2016;355:i5907.

8. Nagendran M, Pereira TV, Kiew G, et al. Very large treatment effects in randomized trials as an empirical marker to indicate whether subsequent trials are necessary: meta-epidemiological assessment.BMJ 2016;355:i5432.

9. Millenson ML. When "patient centred" is no longer enough: the challenge of collaborative health. BMJ,2017;358:j3048. 